\title{
LAS EXPLICACIONES TELEOLÓGICAS Y EL MODELO NOMOLÓGICO-DEDUCTIVO
}

Margarita PONCE

Instituto de Investigaciones Fllosóficas Universidad Nacional Autónoma de Mexico

0. En la raíz del reconocimiento de que las explicaciones teleológicas son genuinas explicaciones, valiosas y útiles para la ciencia y que, además, son irreductibles a las no-teleológicas, se hallan las tesis siguientes: a) ellas revelan uno de los modos básicos en los que opera la razón humana: el que busca la inteligibilidad o la comprensión de los objetos - situaciones, hechos o sucesos - 1 a través de sus resultados o consecuencias. ${ }^{2} \mathrm{Y}, b$ ) en virtud de esta dirección peculiar de nuestros intereses cognoscitivos, las explicaciones teleológicas difieren esencialmente de otros tipos de explicación, por ejemplo, de las causales, que surgen del modo de razonar que trata de comprender los objetos descubriendo los nexos que guardan con algunas de las condiciones antecedentes que los hacen posibles. Ciertamente las tesis anteriores son motivo de controversia, como lo muestra el hecho de que muchos autores que tratan de la teleología, por ejemplo, Beckner, Ruse, Hempel y Bunge, no sólo no las aceptan, sino que aducen toda suerte de argumentos para negar que tales explicaciones posean valor explicativo.

En el presente trabajo examino, precisamente, el argumento que considero decisivo para aceptar o rechazar el carácter explicativo de las explicaciones teleológicas. Este argumento alude a la existencia de alternativas funcionales en los organismos y desemboca en dos posiciones antagónicas: 1) si una misma necesidad vital puede ser satisfecha, indistintamente, de varias maneras o por diversos mecanismos, las explicaciones teleológicas no podrán expresarse como argumentos deductivos, ya que todas las cosas capaces de realizar la misma función deberán estar representadas en la conclusión. Y estas explicaciones tendrán por ello, en el mejor de los casos, sólo un valor heurístico. 2) Si por lo contrario,

1 Para un examen de las razones por las cuales considero que las explicaciones se piden acerca de estados, hechos, o sucesos —entidades extralingüfsticas todas ellasvéase Ponce, M. y Robles, J. A. (1981, pp. 108-10).

2 Por ello Cohen (1978, p. 259) introduce el término "explicación consecuencial" para designar este tipo de explicaciones. 
a partir de un examen cuidadoso de los datos empíricos disponibles, no puede establecerse la existencia de tales alternativas, o dicho de otro modo, si es posible afirmar la existencia única del tipo de objeto funcional (en cada caso), las explicaciones teleológicas resultarán necesarias, lo mismo que las causales, en la tarea de aclarar y sistematizar nuestros conocimientos.

En defensa de (2) y con el fin de enmarcar mejor el problema, explicitaré, en primer término, la caracterización de las explicaciones teleológicas que llamo "estándar". En ella afloran algunos prejuicios muy arraigados sobre la explicación en general y sobre la teleológica en particular; por ejemplo, el de que "explicar" equivale a "explicar causalmente" y el de que las entidades funcionales se explican causalmente por su contribución al logro de fines específicos. Lugo me ocuparé de la naturaleza de la explicación, puesto que las creencias que se tengan sobre ella determinan también nuestro modo de concebir funciones y fines y, por ende, determinan (o restringen) el tipo de argumentos en los que pueden figurar. Finalmente trataré la posible reconstrucción de enunciados funcionales a la luz del modelo nomológico-deductivo de explicación.

1. En la actualidad, no obstante que se hace la distinción entre adscribir propósitos o fines y adscribir funciones, en la literatura científica y filosófica persiste cierta ambigüedad, tanto en el uso de términos teleológicos y funcionales, como en los enunciados y explicaciones en los que aparecen. Por ejemplo, en Teleology Revisited, Nagel afirma que adscribir fines equivale a

enunciar algún resultado o meta hacia el cual ciertas actividades de un organismo o de sus partes se dirigen (1979, p. 277);

así,

El fin del picotear de los pájaros carpinteros consiste en encontrar larvas de insectos (p. 277).

Por otra parte, este mismo autor considera que

las adscripciones de función enuncian cuáles son algunos de los efectos de una cosa o de sus actividades en un organismo... [por ejemplo], la función de las válvulas en el corazón de un vertebrado consiste en proporcionar una dirección a la circulación de la sangre (p. 277). 
Para Wright, sin embargo, la única diferencia entre adscripciones de finalidad y adscripciones de función reside en que

"estar dirigido hacia un fin" es un predicado conductual (Wright 1976, p. 74),

en tanto que no lo es "tiene la función de ..." y, desde la perspectiva de este autor, resulta adecuado afirmar que la oración "el conejo corre para escapar del perro" constituye un ejemplo de adscripción de finalidad, mientras que al adscribir funciones se manejan otros usos de los términos teleológicos que no se refieren directamente a la conducta.

Otra manera de utilizar "finalidad" o "propósito" y "función" es la de Woodfield en Teleology. Para él, aunque toda descripción teleoló gica consiste en adscribir propósitos a las entidades descritas, el uso primario del término "propósito" se da en contextos intencionales. Comúnmente se dice que

el hombre que corre tiene el propósito de alcanzar el tren, [o que] el cuchillo tiene una hoja con el propósito de cortar... (Woodfield 1976, p. 27),

pero señala que según nuestra intuición, la palabra "propósito" se usaría de distinta manera en los ejemplos anteriores, pues sólo sería pertinente hablar de propósitos en tratándose de intenciones. Cosas como los cuchillos no poseen ni unos ni otras. Lo que en realidad hemos hecho es transferir los propósitos de diseñadores y usuarios a los artefactos. En consecuencia, Woodfield propone restringir el uso de "propósito" a contextos intencionales y, en cambio, utilizar la palabra "función" cuando se trate de objetos de los que pueda decirse que las intenciones humanas han plasmado algún propósito particular.

Sin embargo, a pesar de las ambigüedades que ilustran Nagel, Wright o Woodfield (y de otras más), e independientemente del tipo de palabras que se usen en las explicaciones teleológicas, éstas se caracterizan usualmente por la existencia de una doble cadena causal: la primera va de la presencia o la operación de una entidad determinada ("entidad funcional" de aquí en adelante) al estado ("fin") cuya obtención o mantenimiento contribuye a lograr, y la segunda, de esa misma contribución a la presencia, forma u operación de dicha entidad. En las obras de varios autores que se ocupan de la teleología -por ejemplo, en las de Wimsatt, Williams, Ch. Taylor, L. Wright y Woodfield-, encontramos -implícita o explícitamente- esta concepción de las explicaciones teleológicas. De este modo, Wimsatt dice que 
las explicaciones teleológicas [deben considerarse] como explicaciones de la existencia y forma de una entidad funcional, como resultado [el énfasis es mío] del hecho de que ellas contribuyen al logro de propósitos (Wimsatt 1972, p. 11).

En "The Logical Structure of Functional Explanations in Biology", Mary Williams sostiene una concepción similar de este tipo de explicaciones. Según esta autora, el esquema que propone

puede considerarse como la explicitación de la tesis... de que, en biologia, las explicaciones funcionales deberían analizarse como afirmaciones de que el rasgo [la adaptación biológica de que se trate] fue seleccionado en virtud [because] del efecto que se piensa es su función. Las leyes que se utilizan caen dentro de dos conjuntos: [el de] las leyes que establecen la relación causal entre el rasgo y su efecto funcional, y [el de] las leyes que establecen la relación causal entre el efecto funcional y la existencia de dicho rasgo en el sistema (Williams 1976, p. 37).

En sus respectivos análisis de fines y funciones, Charles Taylor (The Explanation of Behaviour) y Larry Wright (Teleological Explanations) afirman que

Ofrecer una explicación teleológica de algún suceso o clase de sucesos, por ejemplo, [de] la conducta de algún ente, es, entonces, dar razón de ella mediante leyes en términos de las cuales se sostiene que la ocurrencia de un suceso depende de que tal suceso sea requerido para algún fin (Taylor 1964, p. 9)

la forma de una explicación teleológica de $\mathbf{B}$ [una muestra de conducta] es: B hace o tiende a hacer esto y aquello, de ahi que B... (Wright 1976, p. 57).

Finalmente, para ilustrar que la caracterización de explicaciones teleológicas, que he denominado "estándar", está fundada en la literatura sobre el tema, citaré lo que sostiene Woodfield:

Las explicaciones funcionales, aun las de artefactos, no dicen meramente 'Esto hace algún bien'; dicen 'Esto está aquí porque hace algún bien'. Las DTs [descripciones teleológicas] funcionales especifican una función y afirman que la función constituye una razón de que la cosa esté allí. Dado que una DT funcional es un enun- 
ciado intrinsecamente explicativo, la palabra "porque" debería figurar en su análisis (Woodfield 1976, p. 136).

Los textos citados revelan que, de las dos cadenas causales discernibles en la caracterización usual de explicaciones teleológicas, por lo menos la segunda - del efecto funcional de alguna entidad a su presencia, forma u operación dentro del sistema en el que se halla- se considera esencial. En consecuencia (como luego veremos), se ha intentado reconstruir estas explicaciones preferentemente como argumentos nomológicodeductivos de tipo causal. Por ello, su aplicación es limitada. En biología, por ejemplo, las explicaciones teleológicas, consideradas conforme a la caracterización estándar, sólo pueden aplicarse de manera plausible al tratamiento de adaptaciones como el color de la piel, el tamaño de ciertos órganos, la operación adecuada de algunos subsistemas, etc.; es decir, adaptaciones cuya determinación se debe a la existencia de varios alelos en la pila génica de la especie, y que pueden favorecer, o no, a individuos de la misma especie. Pero tales explicaciones no pueden aplicarse a otras adaptaciones cuya operación (respecto de la selección natural) pudiera favorecer a algunos individuos sobre otros que pertenezcan a distintos grupos biológicos (como especies, familias o reinos) ${ }^{3}$ del que forman parte dichos individuos más favorecidos.

En fin, habiendo señalado la ambigüedad que persiste en el uso de "fin" y "función", y con el apoyo de los textos citados anteriormente, utilizo aquí la expresión "explicaciones teleológicas" para referirme tanto a adscripciones de función como a adscripciones de finalidad, siempre y cuando los argumentos involucrados recurran a un suceso, hecho o situación posible para explicar un estado de cosas presente.

2. Entre las razones por las que suele negarse que las explicaciones teleológicas sean genuinas explicaciones están, por una parte, las que aducen que con ellas no podemos hacer predicciones adecuadas o que no incrementan nuestra información acerca del mundo. Por otra parte, están las que afirman, o bien que ninguna explicación teleológica puede reconstruirse como argumento nomológico-deductivo, o bien que, en todo caso, no se obtendrá un argumento explicativo de tipo causal.

Considero que las creencias básicas sobre la naturaleza de la expli-

3 Sin entrar aquí en mayor detalle, intuitivamente nos parece correcto afirmar que "la función del color negro en ciertas palomillas de la especie Biston betularia es protegerlas de los pájaros que se alimentan de ellas en áreas contaminadas por el smog" y que este efecto "es causa de que el color negro predomine en la especie". Sin embargo no afirmaríamos que "en virtud de que el hombre tiene manos ha sobrevivido" y que "esto es causa de las manos en los individuos de la especie". 
cación en general son cruciales para la teleología, pues tales creencias pesan decisivamente en la mayoria de los supuestos que atañen tanto al significado de los enunciados funcionales (oraciones de la forma $L a$ función de $\mathrm{X}$ es $Y$ ) como al valor explicativo de las explicaciones funcionales (argumentos con algún enunciado funcional en el explanans). Así, las notables diferencias entré los análisis más representativos sobre este tema, que son los de Nagel y Hempel, se deben con frecuencia a una concepción distinta del papel que desempeña la explicación y también a distintas ideas sobre lo que sea una explicación genuina.

Explicar es hacer inteligible un objeto determinado - que, como señalé antes, puede ser un suceso, un hecho o un estado de cosas expresado en una oración (explanandum) - estableciendo una conexión entre él y alguna otra cosa — también expresada en una oración (explanans) que de algún modo lo determina. En otras palabras, por medio de una explicación llegamos a comprender un objeto en su calidad de efecto o de causa del objeto en cuestión, descrito en el explanans; o bien, lo llegamos a comprender como una entidad compuesta de $x$ partes, o como poseyendo cierta estructura, etc.

En último término he de añadir que establecer la conexión entre el explanandum y el explanans es explicativo porque, implícita o explícitamente, se presupone un principio general que acepta el sujeto que recibe la explicación, y es este principio general el que le confiere validez. Por lo que puede decirse, en palabras de Kim, que las explicaciones "son intentos de exhibir conexiones nomológicas entre sucesos y estados y mostrar cómo caen en patrones legaliformes" (1964, p. 364). Esta función primaria de la explicación se manifiesta a través de un argumento, por lo que también puede decirse que las explicaciones cristalizan en algún tipo de argumento.

Ahora bien, dentro de esta perspectiva, el valor explicativo de un argumento dado no puede medirse en términos de su poder predictivo, ya que una explicación difiere esencialmente de una predicción (o de una retrodicción). Y difiere tanto respecto de las actitudes de las cuales surge como de nuestros propósitos cuando explicamos o predecimos.

Aunque respecto del origen de la explicación no podemos establecer una dicotomía absoluta entre motivos de tipo práctico y otras clases de motivos, sí podemos distinguir diversos tipos de argumento según este criterio. Las razones que impelen al hombre a hacer predicciones son primordialmente prácticas, como la necesidad de sobrevivir en un medio en general hostil y la de controlar los fenómenos de la naturaleza. Para ello, necesita conocer con antelación que, ceteris paribus, se darán ciertos estados de cosas o que ciertos sucesos ocurrirán. De acuerdo con esto y puesto que las predicciones constituyen 
proyecciones de los datos conocidos a los desconocidos... la tarea de un argumento predictivo o retrodictivo es justificar o apoyar tal proyección (Kim, ibid., p. 364).

Antes de proseguir conviene señalar que Kim destaca así el papel de la explicación como parte de las objeciones al tratamiento que hace Hempel de ella, no obstante que este último reconoce: a) que hay preguntas "por qué" que buscan la explicación y preguntas "por qué" planteadas con el propósito de obtener pruebas (o razones) para creer que...; b) que sobre esta base, cuando se da una explicación no se intenta apoyar o demostrar el explanandum, y $c$ ) que la explicación y la predicción difieren en cuanto a ciertos aspectos pragmáticos (Hempel 1965, pp. 334-7). Sin embargo Hempel, cuando se ocupa del valor explicativo de las explicaciones funcionales, las mide con el criterio de su poder predictivo (Hempel 1959, pp. 277-8). Además, distingue entre explicación y predicción tomando en cuenta sólo el tiempo en el cual conocemos el explanans o el explanandum: si el explanans se conoce primero, tenemos un argumento predictivo (o retrodictivo); si, en cambio, conocemos primero el segundo, tenemos entonces una explicación (Hempel 1965, p. 249). Esta distinción de los diversos tipos de argumentos hecha por Hempel, resulta demasiado débil y poco convincente, pues intuimos que debe haber diferencias más profundas entre explicación y predicción, que las relativas sólo al tiempo en el que conocemos las premisas y la conclusión.

En cambio, la búsqueda de explicaciones surge de motivos que no son de índole primordialmente práctica. Entre ellos se cuentan el deseo humano de conocer: según Aristóteles, "Todos los hombres tienen naturalmente el deseo de saber" (Met. 980) y, según Hempel, la raíz de la explicación se halla en "la mera curiosidad intelectual" y en "el profundo y persistente" deseo que tiene el hombre, tanto de comprenderse a sí mismo, como de comprender el mundo en el que vive (Hempel 1965, p. 333). En consecuencia, el propósito de la explicación no consiste principalmente en justificar o demostrar un explanandum por medio de un explanans, sino en aclarar y sistematizar hechos ya conocidos.

Nagel tiene una manera similar de concebir la explicación por lo que, en tratándose del valor de la explicación en general y de la explicación funcional particularmente, sostiene que estas interrogantes

sólo pueden contestarse examinando el papel efectivo que una explicación desempeña en la búsqueda y en la comunicación de las ideas (Nagel 1961, p. 423).

Por lo que toca a las explicaciones funcionales, desde esta perspec- 
tiva resultan explicaciones genuinas y útiles, aunque su función no sea, en general, predecir la presencia o la forma de las entidades funcionales en un sistema determinado. Su función consiste más bien en destacar el papel que dichas entidades desempeñan en la consecución del estado considerado como fin.

Como apunté antes hay, sin embargo, otra manera - representada por Hempel- de concebir la explicación. Esta manera se fija preferentemente en la similitud entre explicaciones y predicciones. $Y$ en ella, el poder predictivo de un argumento se convierte en el criterio para decidir si éste posee o no valor explicativo. Dicho de otro modo, el valor explicativo de un argumento formulado para dar razón de un suceso o hecho particular,

reside en hacer patente que el resultado descrito en el explanandum debía esperarse dadas las circunstancias antecedentes y las leyes generales enlistadas en el explanans. (Hempel 1959, p. 273).

En consecuencia, dentro de esta concepción se confiere a las explicaciones funcionales sólo un valor heuristico, pero no explicativo, aduciendo para ello la razón de que no nos permiten predecir la ocurrencia de una cosa funcional determinada (Cf. Hempel, ibid., pp. 282-7). Más adelante, cuando examinemos el argumento de Hempel para negar que las explicaciones funcionales posean poder predictivo $\mathrm{y}$, por tanto, valor explicativo, veremos que la posición representada por Nagel tiene mayor plausibilidad.

3. Si reflexionamos un poco sobre lo que hemos visto, es claro que tanto las creencias de Hempel sobre la explicación, como la caracterización estándar de las explicaciones teleológicas en términos de dos cadenas causales, constituyen manifestaciones de una arraigada creencia en que el sentido fuerte y genuino de "explicar" equivale a "explicar causalmente": para Hempel, el ideal de la ciencia es obtener una explicación causal de los fenómenos y los enunciados probabilísticos representarían un programa de investigación cuyo objetivo consiste en establecer conexiones causales (Hempel 1965, p. 351). En consonancia con ello, dice que el valor heurístico de las explicaciones teleológicas radica, precisamente, en que contribuyen al descubrimiento de cadenas causales (ibid., p. 330). Y es de notarse que esta afirmación tiene neto sabor aristotélico, pues el Estagirita caracteriza el concepto de explicación mediante la noción de causa: conocemos genuinamente algo cuando conocemos las causas o principios de los cuales depende (Cf. Phys., 194 b 
15-20).4 En nuestros días persiste la creencia de que las nociones de explicación y causa están íntimamente ligadas, a pesar del convencimiento general de que no toda determinación es determinación causal. Muestra de ello son las siguientes afirmaciones de Salmon y de Davidson:

[causas y relaciones causales] pertenecen esencialmente al concepto de explicación (Salmon 1975, p. 128)

[en la tarea de comprender la acción] una creencia y un deseo adecuados podrían explicar, y ser las razones de, una acción sólo si ellos la causan (Davidson 1976, p. 239).

Y debido a que muchos autores que se ocupan de las explicaciones teleológicas -entre ellos Ch. Taylor, L. Wright, H. Lehman, W. Wimsatt y G. A. Cohen- comparten implícita o explícitamente esta concepción de la explicatividad, en los análisis del tema encontramos con frecuencia el intento de reconstruir los enunciados funcionales como argumentos nomológico-deductivos de tipo causal..$^{5}$

A pesar de lo anterior, considero sin embargo que la función esencial de la explicación consiste en volver inteligibles los objetos a explicar y que dicha inteligibilidad puede consistir en comprender el papel que las entidades funcionales desempeñan en un sistema, por lo que es correcto concluir que las explicaciones funcionales son genuinas explicaciones. $\mathrm{Y}$, como toda explicación se expresa mediante un argumento, las explicaciones funcionales también pueden reconstruirse como argumentos de tipo nomológico-deductivo siempre y cuando la descripción del estado considerado el fin del sistema aludido, presente las entidades funcionales como condiciones necesarias para su obtención (o mantenimiento).

4. El argumento clásico para rechazar esta posibilidad es el que señala la presunta existencia de alternativas funcionales en los organismos, las

4 Recordemos que, para Aristóteles, estos principios son cuatro, por lo que la causa eficiente (la causa simpliciter, hoy día) representa en su doctrina sólo una de las maneras posibles en las que puede contestarse la pregunta "por qué."

5 Véase, por ejemplo, Taylor (1964, pp. 9 y 17); Wright (1976, pp. 61, 80, 101); Lehman (1965, p. 19); Wimsatt (1972, pp. 70, 76): Cohen (1978, p. 273). Algunos autores, como Williams, Cohen y Wimsatt, reconocen explicitamente el carácter causal de las explicaciones funcionales, reconstruyéndolas bien sea como argumentos nomológico-deductivos (Williams y Cohen), bien sea como argumentos probabilísticos (Wimsatt). Otros, como Ch. Taylor, señalan que las explicaciones teleológicas son teleologicas en virtud del tipo de leyes que intervienen en ellas, pero, cuando se trata de reconstruirlas, estructuran argumentos deductivos muy similares a los causales. 
cuales servirían para realizar ciertos procesos metabólicos o para satisfacer necesidades vitales. $Y$ las posiciones (antagónicas), clásicas también, ante este problema son las que ejemplifican, de modo ilustre, Nagel y Hempel.

En The Structure of Science, Nagel sostiene que las explicaciones teleológicas difieren de las no-teleológicas tanto a) por el uso de palabras y expresiones peculiares - "fin", "función", "con vista a", "para", etc.-, como (y más importante) b) por los diversos elementos que destacan, a saber, los resultados las teleológicas y las condiciones antecedentes las no-teleológicas. Asimismo cree que $c$ ) las explicaciones teleológicas pueden reformularse, sin pérdida de contenido, como explicaciones no-teleológicas y que, $d$ ) para poderlas reconstruir, los objetos explicados funcionalmente deben describirse en términos de condiciones necesarias (Cf. Nagel 1961, pp. 403, 421-2). De las tesis anteriores, (d) reviste especial importancia porque la validez de los argumentos resultantes depende de si la presencia o actividad de las entidades funcionales se considera o no condición necesaria para lograr el fin. Según Nagel, los enunciados funcionales de la forma general

(I) La función de $A$ en un sistema $S$ con organización $C$ es permitir que $S$ en el medio ambiente $E$ realice el proceso $P$ (Nagel, ibid., p. 403$)$,

que puede ilustrarse con el enunciado:

La función de la clorofila en las plantas es permitir que las plantas realicen la fotosintesis (ibid., p. 403),

son reformulables como enunciados de la forma

(II) Todo sistema $S$ con organización $C$ y en el medio ambiente $E$ realiza el proceso $P$; si $S$ con organización $C$ y en el medio ambiente $E$ no tiene $A$, entonces $S$ no realiza $P$; por tanto, $S$ con organización $C$ debe poseer $A$ (ibid.; p. 403). ${ }^{\circ}$

A la luz de esta reconstrucción, la tesis (c) de Nagel sólo será verdadera si se supone que los efectos funcionales de la entidad a la que se

6 En Teleology Revisited (1979, pp. 314-5) Nagel reformula de nuevo Ios enunciados funcionales de tipo (I). Sin embargo, cito aqui la primera reformulación -(II)por las siguientes razones: la única diferencia entre (II) y la más reciente consiste en que en la última se explicitan con mayor detalle las premisas y la conclusión del argumento; además, las objeciones de Hempel al análisis de Nagel toman en cuenta (II) y no la de Teleology Revisited. 
adscribe la función no constituyen la causa de la presencia o forma de dicha entidad en el sistema. Pero en cambio, si en vez de la suposición anterior, adoptásemos la implicada por la caracterización estándar de explicaciones teleológicas, los argumentos de tipo (II) no podrían considerarse como una traducción adecuada de enunciados de tipo (I), puesto que no conservarían el significado oculto de la palabra "función", que la haría equivalente a "causa de $A$ ". Nagel mismo se da cuenta de este problema y si en The Structure of Science no había especificado aún. el tipo de modelo nomológico deductivo al cual se conforman las explicaciones funcionales, en Teleology Revisited afirma, en cambio, que las adscripciones de función no pueden reconstruirse como explicaciones causales, sino sólo como argumentos nomológico-deductivos, debido a que las funciones que una entidad desempeña no dan razón de ella causalmente ( $C f$. Nagel 1979, p. 315). Como este autor conocía ya las críticas de Hempel a su reconstrucción de enunciados funcionales, allí mismo reitera que los argumentos que propone sí constituyen genuinas explicaciones, pues

hacen evidente un papel que cierta cosa juega en un sistema determinado (ibid., p. 315).

El argumento que aduce en apoyo de estas afirmaciones es, precisamente, que la realización de $P$ (de la fotosíntesis en el ejemplo) no pertenece al conjunto de condiciones antecedentes (no es causa) de la ocurrencia de $A$ (de la clorofila, en el mismo ejemplo). De ahí que, entre las premisas de los argumentos de tipo (II), no haya ninguna ley causal que se refiera al origen de $A$. Conviene aclarar que Nagel consideraría válida esta tesis sólo para las explicaciones funcionales tal como se usan en biología, pues la raíz de su empeño por convencernos de que la reconstrucción de enunciados funcionales no nos da argumentos causales, se halla en que ha captado la diferencia esencial que hay entre las explicaciones de este tipo y las de la acción. Estas últimas sí son causales y por ello distingue ya, también en Teleology Revisited, entre adscribir fines y adscribir funciones; entre la explicación teleológica en sentido estricto, y la funcional (Cf., ibid., pp. 277-8).

5. Es interesante ver cómo la reconstrucción de enunciados funcionales propuesta por Nagel revela dos características peculiares del razonamiento teleológico: por una parte, que las nociones de causa y explicación se relacionan estrechamente en el problema de las explicaciones funcionales o en el de su reformulación no-teleológica, pero no en el sentido de la caracterización estándar de ellas. Así la palabra "debe", 
que aparece en el explanandum del argumento (II), no puede interpretarse como una explicación causal de $A$, sino que es significativa porque sugiere que las entidades funcionales explican causalmente el estado $P$ considerado como fin - tanto en (I) como en (II). Por otra parte, los argumentos de tipo (II), aun si no aparece en ellos ninguna palabra típicamente funcional, pueden considerarse como ejemplos del razonamiento teleológico en el sentido de que ilustran sus etapas: 1) partimos de una situación que nos intriga o nos interesa de alguna manera (el fin) $-P$ en la reformulación de Nagel $-\mathrm{y}$ cuyas condiciones de posibilidad queremos conocer; 2) una vez que, gracias a la investigación empírica, hemos descubierto la causa $-A$ - de esa situación, formulamos entonces el enunciado funcional, que revela nuestra comprensión teleológica de $A$ o sea a través de sus consecuencias, simultánea con nuestra comprensión causal de $P$.

Esta ruta del pensamiento teleológico coincide con la ruta del pensamiento científico, pues en general primero inquirimos por las causas, condiciones antecedentes, condiciones de posibilidad, etc., de una situación específica y luego, al hallar o proponer esas causas, se da la doble comprensión aludida. Raramente se da el caso inverso, es decir, que se encuentre alguna cosa (no buscada a propósito) y luego se formule la pregunta: "Para qué sirve esto?" o "Cuál es la función de esto?" Por todo ello, pienso que la tarea más interesante de los análisis de la teleología consistiría en hacer explícita y destacar su conexión profunda con la causalidad; en enfocar los fines como la clase de efectos que han llamado nuestra atención inquisitiva y las entidades funcionales como sus causas. Así, en este sentido, Woodfield afirma que

El hallazgo principal [en la teleología] es que las funciones son relativas a los fines (Woodfield 1976, p. 100).

Pero, volviendo a la reconstrucción de Nagel, otra consecuencia de ella es que, de acuerdo con el argumento (II), una vez que sabemos qué condiciones son necesarias para que un sistema $-S$ - realice $P$ (para que las plantas realicen la fotosíntesis) también resulta lícito hacer algún tipo de predicción sobre sistemas similares a $S$ (en un medio también similar, etc.), pues concluimos, por ejemplo, que, ceteris paribus, si la fotosíntesis ha de llevarse a cabo, las plantas que la realicen deberán poseer clorofila. Es claro que no se trata aquí de predecir con exactitud la ocurrencia de la entidad funcional en la planta, ni de explicarla causalmente. Se trata más bien de recalcar el papel determinante que desempeña en la consecución del estado-fin.

Ahora bien, respecto a la caracterización estándar de explicaciones 
teleológicas, de acuerdo con lo que hemos visto hasta aquí sobre la naturaleza de la explicación y los argumentos que se derivan de enunciados funcionales, podemos afirmar justificadamente que, para un mejor tratamiento de este tipo de explicaciones, es necesario eliminar la cadena causal que va del efecto funcional de una entidad a su presencia y forma en el sistema en el que se halle. Esta cadena impone límites rígidos a la aplicación de las explicaciones teleológicas e impide considerarlas como la expresión de una de las formas originales e irreductibles del razonamiento humano. En cambio, la cadena causal que va de la ocurrencia de la entidad funcional al estado-fin que contribuye a lograr, no sólo debe permanecer en virtud de que constituye una de las etapas del pensar teleológico, sino que vía esta cadena causal, podemos sostener que las explicaciones teleológicas poseen contenido empírico.

6. Hempel critica la reconstrucción de enunciados funcionales propuesta por Nagel, arguyendo que es formalmente errónea debido a que

el supuesto de la indispensabilidad funcional de un objeto determinado es altamente discutible desde el punto de vista empírico: en todos los casos concretos de aplicación, tal parece que existen otras alternativas; [por ejemplo,] la función de la danza de la lluvia puede ser satisfecha por algún otro ceremonial colectivo (Hempel 1959, p. 284). ${ }^{7}$

Es claro que la tesis (d) de Nagel, o sea, aquélla que, como requisito para reconstruir los enunciados funcionales en forma de argumentos nomológico-deductivos, exige que los objetos a explicar (las entidades funcionales) sean descritos en términos de condiciones necesarias para la realización del estado-fin, constituye el blanco de la objeción de Hempel. Sólo con base en este supuesto, Nagel puede concluir en (II) que

por tanto $S$ con organización $C$ debe poseer $A$.

Y Hempel, quien (como vimos) suele juzgar el valor explicativo de un argumento según su poder predictivo, considera que dada la existencia de alternativas funcionales, es decir, de substancias, procesos o mecanismos alternativos para llevar a cabo la misma función, o para

? Hempel alude en este párrafo, a un ejemplo antropológico de enunciado funcional utilizado por Merton a propósito del ceremonial de la tribu Hopi para propiciar la lluvia, ceremonial cuya función consistirfa en reforzar la identidad del grupo (Hempel 1959, p. 281). 
obtener el mismo estado-fin, la conclusión correcta de los argumentos derivados de enunciados funcionales debería ser

(II') por tanto $S$ con organización $C$ debe poseer o bien $A$, o bien $B$, $o$ bien $C$, etc.

(donde $B, C$, etc., representan dichas alternativas funcionales).

Así, para Hempel, la ocurrencia de alguna entidad funcional sólo puede considerarse como condición suficiente, pero no necesaria, en la consecución de un fin determinado. Propone, pues, un modelo de análisis funcional distinto del de Nagel, que es interesante seguir en detalle: Parte del enunciado

El latir del corazón en los vertebrados tiene la función de hacer que la sangre circule por el organismo (Hempel, ibid., p. 284).

y dice:

El objeto del análisis es cierta "cosa" [item] $i$ que es un rasgo o disposición (por ejemplo, el latir del corazón) relativamente persistente, que ocurre en un sistema $s$ (por ejemplo, el cuerpo de un vertebrado vivo); y el análisis intenta mostrar que $s$ se halla en un estado o condición interna $c_{i}$ y en un medio ambiente que exhibe ciertas condiciones externas $c_{\mathrm{e}}$ tales que, en condiciones $c_{\mathrm{i}}$ y $c_{\mathrm{e}}$ (que conjuntamente serán designadas como $c$ ), el rasgo $i$ tiene efectos que satisfacen cierta "necesidad" o "requerimiento funcional" de $s$, es decir, una condición $n$ que es necesaria para que el sistema permanezca en un funcionamiento adecuado, o efectivo, o conveniente (p. 280). ${ }^{8}$

Basándose en este análisis, Hempel sostiene que las explicaciones funcionales no constituyen ni argumentos deductivos ni inductivos, ya que la existencia de "equivalentes funcionales" las hace ser sólo explicaciones "presuntamente" deductivas. $\mathrm{Y}$ tampoco podrían reconstruirse como argumentos inductivos por la casi-imposibilidad de especificar toda

8 En este análisis, la "cosa $i$ " se refiere a un elemento que es "empíricamente suficiente" para que el requerimiento funcional $n$ sea satisfecho, siempre que: a) "en $t, s$ funcione adecuadamente en un ambiente de clase $c$ "; b) $n$ sea satisfecho para que $s$ funcione adecuadamente, y c) "si el rasgo $i$ estuviese presente en $s$, entonces, como un efecto, la condición $n$ quedaria satisfecha" (cf. Hempel 1965, pp. 310-2). De donde la Clase $I$ estaría constituida por el conjunto de las cosas $i$. Nótese también de paso que Hempel describe aquí las entidades funcionales como algo benéfico para el sistema sobre el que actúan, exponiendo así una tesis que es de neto cuño aristotélico, como puede verse en la Física: el Estagirita sostiene allí que sólo aquel estado que es el mejor para cada ente de naturaleza télica, puede denominarse "fin" (cf. Aristóteles Phys., 194 a. 25-32). 
la gama de patrones de conducta, instituciones, costumbres, [etc.] alternativas, que serían suficientes para satisfacer un prerrequisito o necesidad determinados (Hempel 1959, p. 286).

En consecuencia, el único esquema viable para las explicaciones funcionales sería el siguiente:

(a) En el tiempo $t$, el sistema $s$ funciona adecuadamente dentro de un ambiente de clase $c$.

(b) $s$ funciona adecuadamente en un ambiente de clase $c$ sólo si se satisface la condición $n$.

(c) Alguna de las cosas que pertenecen a la clase I está presente en $s$ en $t$ (ibid., p. 287).

Finalmente este autor concluye que, no existiendo ninguna base, ni inductiva ni deductiva, para esperar que $i_{\varepsilon} I$ y no alguna otra de las alternativas posibles esté presente en $s$, el valor explicativo de las explicaciones funcionales es "precario" y, desde luego, no pueden proporcionar base alguna para hacer predicciones (cf., ibid., p. 288).

7. Los análisis de Nagel y Hempel son decisivos en el tratamiento de la teleología, ya que de su argumentación y resultados depende la suerte de las explicaciones teleológicas como explicaciones genuinas y valiosas para la ciencia. Si Hempel nos ha convencido, mejor nos olvidamos del asunto y nos unimos a quienes opinan (en el mejor de los casos) que el único valor discernible en ellas es el heurístico. Si, en cambio, concedemos que la posición de Nagel es correcta, aunque sea parcialmente, tenemos entonces una buena razón para continuar el examen de la teleología, lo cual haré, pues pienso con este autor que, en la explicación teleológica, las entidades funcionales deben describirse como condiciones necesarias para la consecución del fin. Sin embargo, para sostener esta tesis con mayor plausibilidad, hace falta examinar ciertas dificultades que atañen especialmente al uso de las cláusulas ceteris paribus y al paralelismo entre teleología y causalidad respecto de la descripción de entidades.funcionales y fines, y de la de causas y efectos.

La cuestión central en discusión es la de si existen o no las llamadas "alternativas funcionales" en los organismos. Pues bien, como intentaré justificar ahora, con base en los datos empíricos avalados por la literatura especializada, considero que, estrictamente hablando, tales alternativas no existen.

En primer término, la objeción principal de Hempel al análisis de Nagel es injusta debido a que está hecha desde una perspectiva diferente 
de la de este último. La perspectiva de Hempel es la de ciertas necesidades vitales comunes a todos (o a la mayoría de) los organismos: por ejemplo, la obtención de carbohidratos, la respiración o la "percepción de la luz". Así enfocadas, es verdad que las necesidades funcionales muestran

usualmente, una variedad de soluciones posibles (ibid., p. 285).

Por ejemplo, aunque las plantas sintetizan almidón sólo por medio de la fotosíntesis, los hongos, sin embargo, pueden obtener carbohidratos ingiriendo directamente substancias orgánicas. Hay también varias maneras de realizar el proceso metabólico de la respiración (es decir, de intercambiar gases con el medio ambiente): los peces respiran por las branquias; los anfibios utilizan para ello branquias, piel y pulmones; los mamíferos, sólo los pulmones; en fin, podríamos citar otros muchos ejemplos similares a éstos. Pero, a pesar de todo ello, Nagel tiene razón, pues sus afirmaciones están hechas desde la perspectiva más restringida de ciertas clases especificas de organismos; de organismos que comparten cierta estructura y cierta organización; que se hallan en un medio ambiente también determinado y que funcionan en condiciones normales. Mas, ¿qué entendemos por "condiciones normales"?

Sin explorar en detalle la noción de normalidad, baste decir que "condiciones normales" se refiere aquí a ciertas condiciones ambientales, de estructura, de composición de partes, etc., en las cuales un organismo específico puede funcionar naturalmente de manera adecuada, como el pez en el agua, un mamífero con su propio y original corazón, etc. Así, por ejemplo, las focas pueden permanecer funcionando bien bajo la superficie del agua sólo por corto tiempo. Si el tiempo de inmersión se prolonga, el organismo de estos animales comienza a trabajar en una situación de emergencia o anormal. En situaciones anormales, ciertamente otros mecanismos - que involucran a veces órganos distintos, o substancias diferentes - satisfacen la misma necesidad vital o cumplen la misma función; de este modo, las focas pueden sobrevivir (dentro de ciertos límites) en esa situación de emergencia; su organismo respira entonces anaeróbicamente pero sin duda, en ellas, este tipo de respiración no puede considerarse como una genuina alternativa funcional, ya que es menos eficiente que la aeróbica (cf. Scholander 1963). Por lo menos intuitivamente, no parece que las expresiones "alternativa funcional" y "dispositivo de emergencia" sean equivalentes. Ahora bien, si tomamos en cuenta las restricciones anteriores, podemos afirmar que en los organismos particulares sólo hay una manera adecuada y eficiente de realizar las funciones vitales, por lo que, desde el punto de vista del 
equipo natural de un ser vivo y de la eficacia en la satisfacción de sus necesidades, la entidad funcional se manifiesta como una condición necesaria para mantener $\mathbf{u}$ obtener el estado-fin. $Y$ esto, como atinadamente observa Nagel, no sería incompatible con el hecho de que la entidad funcional en cuestión estuviese constituida por un conjunto de elementos (que pueden ser o no similares), por ejemplo las dos orejas, que cumplen la misma función, o alguno de los sistemas homeostáticos del cuerpo. (cf. Nagel 1979, p. 308).

En cuanto al hecho de que en ciertas ocasiones una misma función puede ser satisfecha en un mismo organismo por mecanismos artificiales diferentes, pienso que, con base en tal hecho, no podemos aducir ninguna buena razón para no describir las entidades funcionales en términos de condiciones necesarias. Aludiendo a situaciones comunes en nuestro tiempo, la circulación de la sangre, por ejemplo, puede llevarse a cabo tanto por la operación del corazón como por la de una bomba mecánica. Sin embargo, no por ello dejaremos de afirmar que el latir del corazón representa una condición necesaria para que la sangre de los vertebrados circule, pues constituye una operación natural (y por tanto forma parte de las condiciones normales) en (de) ellos; mientras que una bomba mecánica - aún suponiendo que ya se pudiese implantar por varios años- es un dispositivo de emergencia y, por ende, anormal.

8. La dificultad a la que aludí antes acerca de las cláusulas ceteris paribus se manifiesta ya claramente: por una parte, parece plausible reconstruir los enunciados funcionales como argumentos nomológicodeductivos, puesto que el argumento de Hempel en favor de que las entidades funcionales se consideren sólo como condiciones suficientes no es conclusivo - según los datos científicos disponibles hasta ahoraPero, por otra parte, el costo de tal reconstrucción parece excesivo, pues podria argüirse que la explicación obtenida finalmente, en virtud de las rígidas restricciones concernientes a las condiciones normales de funcionamiento de una clase específica de organismos, es trivial y de escaso contenido empírico.

En defensa de la teleología, diré que esta dificultad no atañe únicamente a las explicaciones teleológicas, sino que en ellas aflora el problema general del uso de las cláusulas ceteris paribus. Es cierto que, si relativizamos en exceso nuestras descripciones a una serie de factores como los señalados, corremos el riesgo de obtener argumentos y definiciones ad hoc. Pero tampoco podemos prescindir de dichas cláusulas, pues si dejásemos de considerar los sistemas (físicos, biológicos, sociales, etc.) que constituyen nuestros objetos de estudio, como sistemas aislados 
(dentro de ciertos límites, por supuesto), nos perderíamos en un conjunto infinito de interacciones universales y no podriamos formular ningún tipo de explicación sobre ellos. Sin cláusulas ceteris paribus la ciencia se convertiría en una empresa imposible, por lo que ellas son necesarias -y a la vez poco atractivas - tanto en el razonamiento teleológico como en el no-teleológico.

9. La reconstrucción de enunciados funcionales conlleva también un problema común a la teleología y a la causalidad: el de la descripción de fines y entidades funcionales en la primera, y el de la descripción de causas y efectos en la segunda. Las categorías de necesidad y suficiencia son relativas a lo adecuado y completo de nuestras descripciones y, por ende, son importantes en el plano explicativo más que en el ontológico. Sólo si describimos fines y efectos de manera adecuada podemos considerar las entidades funcionales y las causas como condiciones necesarias para la producción de los primeros. Así, Davidson destaca esa simetría en la descripción de las causas como suficientes o necesarias y la incompletud o completud de la descripción de los efectos (cf. Davidson 1967, p. 89). Y como era de esperarse, también Nagel es sensible a este problema -cuya resolución es decisiva para su tesis sobre la manera de reconstruir las explicaciones funcionales-. En consecuencia, señala la conexión tan estrecha que hay entre causalidad y teleología respecto de los términos "necesidad" y "suficiencia" ( $c f$. Nagel 1979, pp. 307-8). Para ello, trae a colación un ejemplo clásico al que recurren tanto los que sostienen que las causas no pueden considerarse como condiciones necesarias para sus efectos $\longrightarrow$ bien, trasladando la cuestión al terreno ontológico, que un mismo efecto puede tener varias causas--, como los que sostienen que las entidades funcionales son sólo condiciones suficientes para obtener el fin, a saber, que la muerte puede ser resultado de múltiples causas, entre ellas, ahogamiento, heridas de arma de fuego, veneno, etc. (cf. Bunge 1972, p. 136.) Este ejemplo convence superficialmente debido a que la tesis subyacente en él parece plausible

sólo porque las causas de la muerte se han analizado más precisamente y se las ha clasificado mejor, que la manera en la que esto se ha hecho con sus efectos (Nagel, ibid., p. 307).

Piensa Nagel que, para presentar las entidades funcionales como condiciones necesarias en la explicación teleológica, se requiere que los términos que aparecen en ella sean descritos adecuadamente. Así, en relación con el caso de la muerte y sus múltiples causas posibles, este autor 
hace notar que, si comparamos el cuerpo de alguien cuya muerte haya ocurrido por ahogamiento, con el cuerpo de alguien cuya muerte haya tenido otra causa, encontraremos grandes diferencias entre uno y otro, por lo que sus respectivas descripciones diferirán también. ${ }^{\circ}$ En términos ontológicos podría decirse que cada tipo de causa produce un efecto peculiar, lo cual es corroborado por la evidencia empírica, pues en cualquier tratado de fisiología vemos que a un efecto peculiar corresponde una causa específica. Así, volviendo al ejemplo anterior, no sólo resulta que la descripción adecuada del cuerpo de quien murió por ahogamiento difiere de la del cuerpo de quien murió por otras causas, sino que aun diferirán entre sí las descripciones de los cuerpos de quienes mueren ahogados, según ello ocurra en agua salada, en agua dulce, etc. (cf. Guyton 1971, p. 575.) Es decir - y vale la reiteración- que los datos científicos inclinan la balanza en favor de la tesis de que, mientras mejor sea nuestra descripción del efecto-fin, la causa-entidad funcional podrá considerarse con mayor plausibilidad como condición necesaria de su ocurrencia. Por tanto, es factible reconstruir enunciados funcionales a la manera de Nagel, como argumentos nomológico-deductivos, manera que, en principio, garantiza el valor explicativo y el contenido empírico de las explicaciones teleológicas.

10. En conclusión, después de lo que hemos visto, me parece lícito afirmar que las explicaciones teleológicas son genuinas, útiles a la ciencia e irreductibles a otro tipo de explicación. Esta tesis se funda, por una parte, en el examen de la naturaleza de la explicación en general y, por otra, en el examen de ciertos datos empíricos que supuestamente apoyarían la afirmación de que, en los organismos, existen alternativas funcionales.

Del examen de la naturaleza de la explicación, concluimos que: a) la función primaria de ésta no consiste en hacer predicciones sino en volver inteligibles los sucesos o situaciones que deseamos explicar; $b$ ) esta inteligibilidad se obtiene por varias vías: o bien estableciendo conexiones causales entre el objeto por explicar y aquello que lo explica, o bien relaciones de otro tipo, como las consecuenciales -es decir, en este último caso, que los objetos en cuestión se comprenden como la causa (o parte de la causa) de cierto estado-fin, y $c$ ) las conexiones así establecidas son válidas gracias a que suponemos que hay principios o leyes

9 En contra de la tesis de que las entidades funcionales deben considerarse sólo como condiciones suficientes del estado-fin (es decir, contra el análisis de Hempel de las explicaciones funcionales), Nagel dice -refiriéndose al ejemplo hempeliano del latir del corazón- que negar que el corazón sea necesario para la circulación, indica una manera laxa o poco precisa de usar el término "cuerpo humano" (o, para el caso, "el sistema $S$ ") (Nagel, ibid., p. 307). 
generales de los cuales nuestro explanandum - por ejemplo, la ocurrencia de una determinada entidad funcional - es un caso particular o, dicho de otro modo, en la explicación se subsume el explanandum bajo un principio general con ayuda de las condiciones especificadas en el explanans-, por lo que las explicaciones deben poderse expresar como algún tipo de argumento.

Aunque, las explicaciones teleológicas sean genuinas explicaciones según los criterios anteriores, este carácter se les ha negado (como ya he señalado) aduciendo que: ( $\left.a^{\prime}\right)$ carecen de poder predictivo, $\left(b^{\prime}\right)$ en la mayoría de los casos, la ocurrencia de la entidad funcional en un sistema no puede explicarse causalmente en virtud de su contribución al logro o mantenimiento del fin, y $\left(c^{\prime}\right)$ los enunciados funcionales no son reconstruibles como argumentos ni deductivos ni inductivos. Ahora bien, (a) y (b) proporcionan buenas razones para sostener que las objeciones $\left(a^{\prime}\right)$ y $\left(b^{\prime}\right)$ surgen de una concepción equivocada de la naturaleza de la explicación. En cuanto a ( $\left.c^{\prime}\right)$, el examen de las pruebas empíricas pertinentes nos autoriza a negar la existencia de alternativas funcionales en organismos de una misma clase $y$, como esa presunta existencia fundaría la tesis de que las explicaciones teleológicas no son expresables ni en argumentos inductivos ni deductivos, concluimos asimismo que esta objeción $-\left(c^{\prime}\right)$ - es infundada.

Por último, insistiré en señalar que la caracterización estándar de las explicaciones teleológicas es inadecuada porque en ella subyace la idea de que sólo las explicaciones causales son dignas de tal nombre, idea que cristaliza en el requisito de que los efectos funcionales de una entidad expliquen causalmente la presencia de ella en un sistema. Así, caracterizar las explicaciones teleológicas de este modo se contrapone a la condición (b) de la explicación y, además, limita el campo de aplicación de ellas al reducido ámbito de las adaptaciones biológicas cuyo origen se halla en la existencia de varios alelos —en la pila génica de la especie- que determinan un mismo rasgo fenotípico.

\section{REFERENCIAS BIBLIOGRÁFICAS}

Aristotle, Metaphysica (Trad. W. D. Ross), en The Basic Works of Aristotle. Random House, New York, 1941.

- Physica (Trad. R. P. Hardie y R. K. Gaye), en The Basic Works of Aristolle. Random House, New York, 1941.

Beckner, Morton (1976), "Function and Teleology", en Topics in the Philosophy of Biology. D. Reidel Publ. Co., Dordrecht, Holland, 1976.

Bunge, Mario, Causalidad (Trad. Hernán Rodríguez) (3ạ ed.). Editorial Universitaria de Buenos Aires, Argentina, 1972. 
Cohen, G. A. (1978) Karl Marx's Theory of History. Clarendon Press, Oxford. Davidson, Donald (1976) "Hempel on Explaining Action", Erkenntnis, Vol. 10, pp. 239-253, D. Reidel Publ. Co. Dordrecht-Holland.

(1967), "Causal Relations" (82-94), en Sosa, Ernest (Ed), Causation and Conditionals. Oxford University Press, London, 1975.

Guyton, Arthur C. (1971), Tratado de Fisiologia Médica (Trad. Alberto Folch y Pi). Nueva Editorial Interamericana, México.

Hempel, Carl, G. (1959), "The Logic of Functional Analysis", en Gross Llewellyn (ed.),Symposium of Sociological Theory. Harper and Row, New York.

- (1965) Aspects of Scientific Explanation and Other Essays in the Philosophy of Science. The Free Press, New York, Collier-McMillan Limited, London. Kim, Jaegwon (1964), "Inference, Explanation and Prediction", en Journal of Philosophy. Vol. 61, pp. 360-368.

Lehman, Hugh (1965), "Functional Explanation in Biology", en Philosophy of Science. Vol. 32, no 1.

Nagel, Ernest (1961), The Structure of Science. Harcourt Brace and World, Inc. U.S. A.

- (1979) Teleology Revisted. Columbia University Press, U.S. A.

Ponce, Margarita (1980), "Aristóteles y la teleología actual", Diánoia 1979. F. C. E., México, pp. 101-25.

Ponce, Margarita y Robles, José A. (1981), "Notas generales sobre la explicación", Diánoia 1980, F. C. E., México, pp. 105-33.

Salmon, Wesley (1975), "Theoretical Explanation", en Stephan Körner (ed.), Explanation, Yale University Press, New Haven.

Scholander, P. F. (1963), "The Master Switch of Life", W. H. Freeman and Co. Tomado de Scientific American, Dic. 1963.

Taylor, Charles (1964), The Explanation of Behaviour. Routledge and Kegan Paul Ltd., London, 1965. 2nd. ed.

Williams, Mary B. (1976), "The Logical Structure of Functional Explanations in Biology", en PSA. Vol. I, pp. 37-46.

Wimsatt, William C. (1972), 'Teleology and the Logical Structure of Function Statements", en Studies in History and Philosophy of Science 3 no 1, Great Britain.

Woodfield, Andrew (1976), Teleology. Cambridge University Press, Great Britain.

Wright, Larry (1976), Teleological Explanations. University of California Press, U. S. A. 